\title{
On group-theoretic models of randomness and genericity
}

\author{
Ilya Kapovich and Paul Schupp*
}

\begin{abstract}
We compare Gromov's density model of random groups with the ArzhantsevaOl'shanskii model of genericity.
\end{abstract}

Mathematics Subject Classification (2000). 20F69; 20F65, $20 \mathrm{E} 07$.

Keywords. Genericity, random groups, small cancellation groups.

\section{Introduction}

The idea of genericity in geometric group theory was suggested by Gromov and Ol'shanskii in late 1980s. This theme has become the subject of active study in recent years.

The first mention of the idea of group-theoretic genericity seems to have been made in a 1986 paper of Guba [15]. The first definition of genericity in the context of finitely presented groups is due to Gromov and appeared in his seminal 1987 monograph "Hyperbolic groups" [12]. There Gromov stated that for any fixed $k \geq 2$ and $m \geq 1$ we have

$$
\lim _{\min n_{i} \rightarrow \infty} \frac{N_{h}\left(k, m, n_{1}, \ldots, n_{m}\right)}{N\left(k, m, n_{1}, \ldots, n_{m}\right)}=1 .
$$

Here $N\left(k, m, n_{1}, \ldots, n_{m}\right)$ is the number of all finite presentations of the form

$$
\left\langle a_{1}, \ldots, a_{k} \mid r_{1}, \ldots, r_{m}\right\rangle
$$

where $r_{i}$ are cyclically reduced words with $\left|r_{i}\right|=n_{i}$ for $i=1, \ldots, m$ and $N_{h}\left(k, m, n_{1}, \ldots, n_{m}\right)$ is the number of those among such presentations that define word-hyperbolic groups. Later Ol'shanskii [30] and Champetier [6], [7] gave rigorous proofs of this result.

* Both authors were supported by the NSF grant DMS\#0404991. The first author was also supported by the NSF grant DMS\#0603921 
The second model of genericity, which we term the Arzhantseva-Ol'shanskii model, suggested by Ol'shanskii in 1989 in a problem that appeared in the 11th edition of Kourovka Notebook [22] (problem 11.75 in [22] contains a notion that is very similar to, but slightly different from, the definition of Arzhantseva-Ol'shanskii genericity used in [1], [5], [18] and in the present paper).

A property $\mathcal{P}$ of finitely presented groups is generic in the Arzhantseva-Olshanskii model (correspondingly, exponentially generic if the convergence to 1 in the limit below is exponentially fast) if for every $k \geq 2, m \geq 1$ we have

$$
\lim _{n \rightarrow \infty} \frac{\beta_{\mathcal{P}}(k, m, n)}{\beta(k, m, n)}=1 .
$$

Here $\beta(k, m, n)$ is the number of presentations of the form $(*)$ where $\max _{i}\left|r_{i}\right| \leq n$ and $\beta_{\mathcal{P}}(k, m, n)$ is the number of such presentations that define a group with property $\mathcal{P}$. We will give more precise definitions related to this model in Section 3 below. The Arzhantseva-Ol'shanskii model is somewhat easier to work with than Gromov's original model, since one can essentially disregard the situation where some defining relators are much shorter than others. This second model of genericity was formally introduced by Arzhantseva and Ol'shanskii [5] where they proved that the property of a $k$-generated $m$-related group to have all $(k-1)$-generated subgroups being free is exponentially generic for every $k \geq 2, m \geq 1$. The Arzhantseva-Ol'shanskii model was subsequently used by Arzhantseva [1], [2], [3], [4] and, later, by the authors of this paper [18], [19], [21], [20]. For example, Kapovich, Schupp and Shpilrain [21] discovered a phenomenon of Mostow-type isomorphism rigidity for generic one-relator groups using the Arzhantseva-Ol'shanskii model.

In his book "Asymptotic invariants of infinite groups" [13], Gromov introduced another model of genericity that we refer to as Gromov's density model of random groups. In this model one first fixes a density parameter $0<d<1$. Then, given a number of generators $k \geq 2$ and an integer $n>>1$, from the set of all cyclically reduced words of length $n$ in $F\left(a_{1}, \ldots, a_{k}\right)$ one chooses uniformly randomly and independently $(2 k-1)^{d n}$ elements forming a set $R$. Here $F\left(a_{1}, \ldots, a_{k}\right)$ denotes the free group with free basis $\left\{a_{1}, \ldots, a_{k}\right\}$. The group

$$
G=\left\langle a_{1}, \ldots, a_{k} \mid R\right\rangle
$$

is termed a random group with density parameter $d$ or a $d$-random group. One then tries to understand the properties of $G$ as $n \rightarrow \infty$. Note that the number of defining relators $(2 k-1)^{d n}$ grows exponentially in the length $n$ of the relators. Also, crucially, the density parameter $d$ does not depend on the number of generators $k$ of $G$. Gromov's density model was further explored by Ollivier [23], [24], [25], [26], Zuk [33] (who used a "triangulated" variation of this model), Ollivier-Wise [28], [29], and others. Thus Ollivier [23], [24], [27] gave a precise proof (with some generalizations to the case of random quotients of word-hyperbolic groups) of a result first outlined 
by Gromov that for $d<1 / 2$ a $d$-random group is non-elementary torsion-free wordhyperbolic and for $d>1 / 2$ a $d$-random group is finite (in fact either trivial or cyclic of order two).

In [14] Gromov used yet another model of randomness, which one might call a random graphical quotient model, to prove the existence of a finitely generated group that does not admit a uniform embedding into a Hilbert space. Ghys [11] gives an exposition of the results and ideas related to Gromov's density and graphical models of genericity. A subsequent survey of Ollivier [27] gives a more updated presentation of these topics.

Yet another approach to genericity involves considering the space $\widetilde{S}_{k}$ of marked groups (that is, the space of normal subgroups $N$ in a fixed free group $F\left(a_{1}, \ldots, a_{k}\right)$ or, equivalently, the quotients $\left.F\left(a_{1}, \ldots, a_{k}\right) / N\right)$ as a topological space. One can then take the closure of some interesting class of finitely presented groups (e.g. of word-hyperbolic groups) and try to understand the algebraic properties of typical groups contained in this closure. This approach was explored, in particular, by Champetier [8].

Our goal in this paper is to clarify the relationship between Gromov's density model and the Arzhantseva-Olshanskii model. While there is no direct connection between them, it turns out that proofs using the Arzhantseva-Ol'shanskii model often imply that a certain variation of Gromov's density randomness condition holds.

For the purposes of comparison we need to introduce a variant of Gromov's density model of randomness where the density parameter $d=d(k)$ depends on the number of generators $k$ and where it is possible that $d(k) \rightarrow 0$ as $k \rightarrow \infty$. We call this notion low-density randomness (see Section 2 for precise definitions, including the definition of a monotone low-density random property). We show in Theorem 5.4 that many algebraic genericity results obtained in the Arzhantseva-Ol'shanskii model do yield low-density random properties:

Theorem 1.1. The following properties are monotone low-density random (where $k$ varies over $k=2,3, \ldots)$ :

(1) the property that a finite group presentation defines a group $G$ that is one-ended, torsion-free and word-hyperbolic [5], [1] (in fact, this property is monotone random in Gromov's density model [14], [24]).

(2) the property that a finite presentation on generators $a_{1}, \ldots, a_{k}$ defines a group $G$ such that all $(k-1)$-generated subgroups are free and quasiconvex in $G$;

(3) the property that a finite presentation on generators $a_{1}, \ldots, a_{k}$ defines a group $G$ with $\operatorname{rk}(G)=k$.

(4) the property that a finite presentation on generators $a_{1}, \ldots, a_{k}$ defines a group $G$ such that all $L_{k}$-generated subgroups of infinite index in $G$ are free and quasiconvex in $G$ (here $L_{k}$ is any sequence of positive integers). 
(5) the property that for a $k$-generated finitely presented group $G$ there is exactly one Nielsen-equivalence class of $k$-tuples of elements generating non-free subgroups.

Recall that for a finitely generated group $G$ the $\operatorname{rank}$ of $G$, $\operatorname{denoted} \operatorname{rk}(G)$, is the smallest cardinality of a generating set for $G$.

It turns out that in many cases various properties that are generic in the Arzhantseva-Ol'shanskii model are not $d$-random in the sense of Gromov with $d$ independent of $k$. Some key information for estimating the density parameter $d$ in Gromov's model is contained in the genericity entropy of exponentially generic sets of cyclically reduced words in $F\left(a_{1}, \ldots, a_{k}\right)$. The definition of exponential genericity for subsets of $F\left(a_{1}, \ldots, a_{k}\right)$ requires that certain fractions converge to 1 exponentially fast as $n \rightarrow \infty$. Genericity entropy quantifies this convergence rate.

We observe here that, unlike the standard small cancellation conditions, for the Arzhantseva-Ol'shanskii "non-readability condition" the genericity entropy depends on the number of generators $k$ and in fact converges to 1 as $k \rightarrow \infty$. This implies that, when translated into the language of Gromov's density model, various results using the Arzhantseva-Ol'shanskii model yield properties that are low-density random but which are not $d$-random for any fixed $d>0$ which is independent of $k$. We prove this fact in detail (see Corollary 6.2 below) for the Arzhantseva-Ol'shanskii non- $\mu$ readability condition. We also show (see Proposition 6.4 below) that the property for a finite presentation on $k$ generators to define a group $G$ with $\operatorname{rk}(G)=k$ is low-density random but not $d$-random for any $d>0$ independent of $k$. The same is true (see Corollary 6.3 below) for the analog of Magnus' Freiheitssatz, that is, for the property that for a group $G$ defined by a finite presentation on the generators $a_{1}, \ldots, a_{k}$, any proper subset of $a_{1}, \ldots, a_{k}$ freely generates a free subgroup of $G$.

We show, however, that certain results obtained in the Arzhantseva-Ol'shanskii genericity model do yield $d$-random properties in Gromov's sense. Thus we prove (see Theorem 7.5 below):

Theorem 1.2. For any fixed integer $L \geq 2$ there is some $d_{L}>0$ such that the property that all L-generated subgroups of infinite index in a finitely presented group $G$ are free is monotone $d_{L}$-random.

We also apply our results to a question of estimating from below the number of isomorphism types of quotients of $F\left(a_{1}, a_{2}, \ldots, a_{k}\right)$ where the number of relators is arbitrary and their length is bounded above by $n$. To be more precise, let $k \geq 2$ be fixed and let $I_{k}(n)$ be the number of isomorphism types of groups given by presentations of the form

$$
\left\langle a_{1}, \ldots, a_{k} \mid R\right\rangle
$$

where $R$ is a subset of the $n$-ball in $F\left(a_{1}, \ldots, a_{k}\right)$. Note that the size of the $n$ ball in $F\left(a_{1}, \ldots, a_{k}\right)$ is $\leq(2 k-1)^{n}$. Hence the number of all subsets of this ball 
is $\leq 2^{(2 k-1)^{n}}$ yielding a double-exponential upper bound on $I_{k}(n)$ as $n \rightarrow \infty$. It is natural to ask if there is also a double-exponential lower bound for $I_{k}(n)$. This question was suggested to the authors by Gromov, who informed us that several years ago Anna Erschler obtained an unpublished proof giving such a double-exponential lower bound. In this paper, relying on the isomorphism rigidity results for generic quotients of the modular group that we obtained in [20], we achieve a double-exponential lower bound for $I_{k}(n)$. Our proof is quite different from that of Erschler who used central extensions of word-hyperbolic groups to estimate $I_{k}(n)$ from below.

Let $M=\left\langle a, b \mid a^{2}=b^{3}=1\right\rangle$, so that $M$ is isomorphic to the modular group $\operatorname{PSL}(2, \mathbb{Z})$. We consider finitely presented quotients of $M$ where the defining relations are words in the alphabet $A=\left\{a, b, b^{-1}\right\}$. There are natural notions of a reduced and a cyclically reduced word in $A^{*}$ in this setting (see Section 8 below for details). Note that in this context every cyclically reduced word is either a single letter or has even length (again, see Section 8 below). Let $\epsilon>0$ be fixed. For an integer $t \geq 1$ let $J_{\epsilon}(t)$ be the number of isomorphism types of groups given by presentations of the form

$$
G=M /\left\langle\left\langle r_{1}, \ldots, r_{m}\right\rangle\right\rangle
$$

where $m=2^{t \epsilon}$ and where each $r_{i}$ is a cyclically reduced word of length $2 t$ in $A^{*}$.

Theorem 1.3. There exists $\epsilon_{0}>0$ such that for any $0<\epsilon \leq \epsilon_{0}$ there is some $\rho>1$ such that

$$
J_{\epsilon}(t) \geq \rho^{\rho^{t}} \text { for } t \rightarrow \infty,
$$

that is, the number $J_{\epsilon}(t)$ is bounded below by a double-exponential function of $t$ as $t \rightarrow \infty$.

Since $M$ is generated by two elements $a$ and $b$, Theorem 1.3 immediately yields a double-exponential Erschler lower bound:

Corollary 1.4. The function $I_{2}(n)$ has a double-exponential lower bound (and hence the same is true for $I_{k}(n)$ for any fixed $k \geq 2$ ).

We are grateful to Lior Silberman for helpful comments regarding the behavior of Kazhdan's property (T) with respect to Gromov's density model. We also thank Goulnara Arzhantseva for many helpful remarks and suggestions.

\section{Gromov's density model and low-density random groups}

In this section we want to give some precise definitions and notation related to Gromov's density model. 
Notation 2.1. For $k \geq 2$ let $\ell_{k} \subseteq F\left(a_{1}, \ldots, a_{k}\right)$ be the set of all cyclically reduced words in $F\left(a_{1}, \ldots, a_{k}\right)$. If $\mathcal{P}_{k} \subseteq \mathcal{C}_{k}$, we denote $\overline{\mathcal{P}_{k}}:=\mathscr{C}_{k}-\mathcal{P}_{k}$. For a subset $\mathcal{Q}_{k} \subseteq F\left(a_{1}, \ldots, a_{k}\right)$ denote by $\gamma\left(n, \mathcal{Q}_{k}\right)$ the number of elements of length $n$ in $\mathcal{Q}_{k}$.

Definition 2.2 (Random groups in the density model). Let $\mathcal{E}$ be a property of finite presentations of groups. Let $0<d<1$.

We say that the property $\mathcal{G}$ is random with density parameter $d$ (or $d$-random) if for every $k \geq 2$

$$
\lim _{n \rightarrow \infty} \frac{R_{k}(n, d, \mathscr{E})}{\gamma\left(n, \mathscr{C}_{k}\right)^{m_{n}}}=1,
$$

where $m_{n}=(2 k-1)^{d n}$ and $R_{k}(n, d, \mathscr{E})$ is the number of all $m_{n}$-tuples $\left(r_{1}, \ldots, r_{m_{n}}\right)$ of cyclically reduced words of length $n$ such that the group with presentation

$$
\left\langle a_{1}, \ldots, a_{k} \mid r_{1}, \ldots, r_{m_{n}}\right\rangle
$$

has property $\mathcal{G}$.

We say that $\mathcal{E}$ is monotone $d$-random if for every $0<d^{\prime} \leq d$ the property $\mathscr{E}$ is $d^{\prime}$-random. A property is monotone random of it is monotone $d$-random for some $d>0$.

Note that $\gamma\left(n, \mathscr{C}_{k}\right)^{m_{n}}$ is exactly the number of all presentations

$$
\left\langle a_{1}, \ldots, a_{k} \mid r_{1}, \ldots, r_{m_{n}}\right\rangle
$$

where the $r_{i}$ are cyclically reduced words of length $n$.

Definition 2.3 (Low-density random groups). We consider a property $\mathcal{G}$ of finite presentations as $\mathscr{E}=\left(\mathscr{E}_{k}\right)_{k \geq 2}$ where, for every $k \geq 2, \boldsymbol{E}_{k}$ is a property of finite group presentations on $k$ generators $a_{1}, \ldots, a_{k}$.

For every integer $k \geq 2$ let $0<d(k)<1$. We say that $\mathscr{E}$ is low-density random with density sequence $(d(k))_{k \geq 2}$ if for every integer $k \geq 2$ we have

$$
\lim _{n \rightarrow \infty} \frac{R_{k}\left(n, d(k), \mathscr{e}_{k}\right)}{\gamma\left(n, \mathscr{C}_{k}\right)^{m_{n}}}=1,
$$

were $m_{n}=(2 k-1)^{n d(k)}$ and $R_{k}\left(n, d(k), \boldsymbol{g}_{k}\right)$ is the number of all $m_{n}$-tuples $\left(r_{1}, \ldots, r_{m_{n}}\right)$ of cyclically reduced words of length $n$ such that the group

$$
\left\langle a_{1}, \ldots, a_{k} \mid r_{1}, \ldots, r_{m_{n}}\right\rangle
$$

has property $\mathscr{E}_{k}$.

We say that $\mathcal{G}$ is monotone low-density random with density sequence $(d(k))_{k \geq 2}$ if for any sequence $\left(d^{\prime}(k)\right)_{k \geq 2}$ satisfying $0<d^{\prime}(k) \leq d(k)$ the property $\mathscr{E}$ is lowdensity random with density sequence $\left(d^{\prime}(k)\right)_{k \geq 2}$. 
Remark 2.4. In the above definition let $d:=\inf _{k} d(k)$ and let $\mathscr{E}$ be monotone low-density random with density sequence $(d(k))_{k \geq 2}$. If $d>0$ then $\mathscr{E}$ is monotone $d$-random in the sense of Definition 2.2.

The situation where $d=0$ does not, however, correspond to a special case of Definition 2.2.

Note that if $\mathcal{G}$ is a monotone low-density random property and $\mathscr{U}^{\prime}$ is a monotone random property with a density parameter $d>0$ independent of $k$, then $\mathscr{E} \cap \mathcal{E}^{\prime}$ is again monotone low-density random. Moreover, the intersection of two monotone low-density random properties is also monotone low-density random.

In this paper we concentrate on monotone random and monotone low-density random properties. There are, however, important examples of non-monotone random properties. Thus it follows from the result of Zuk [33] that Kazhdan's property (T) is $d$-random for every $1 / 3<d<1 / 2$ (Zuk uses a somewhat different density model in his paper, but his results imply the above statement in Gromov's density model). On the other hand, Ollivier and Wise [28] proved that if $0<d<1 / 5$ and $G$ is a $d$-random group, then $G$ does not have property (T).

\section{The Arzhantseva-Ol'shanskii genericity model}

We recall the basic notion of genericity in the Arzhantseva-Ol'shanskii approach.

Definition 3.1 (Generic subsets). Let $k \geq 2$ be an integer. A subset $\mathcal{P}_{k} \subseteq \mathscr{C}_{k}$ is generic if

$$
\lim _{n \rightarrow \infty} \frac{\gamma\left(n, \mathcal{P}_{k}\right)}{\gamma\left(n, \mathscr{C}_{k}\right)}=1
$$

We say that $\mathcal{P}_{k} \subseteq \mathcal{C}_{k}$ is exponentially generic if it is generic and, in addition, the convergence to 1 in the above limit is exponentially fast, that is, there exist $a>0$ and $0<\sigma<1$ such that

$$
\frac{\gamma\left(n, \overline{P_{k}}\right)}{\gamma\left(n, \mathscr{C}_{k}\right)} \leq a \sigma^{n}
$$

for all $n \geq 1$. This condition is equivalent to the fact that for some $0<t<1$ and some $c>0$ we have

$$
\gamma\left(n, \overline{\mathcal{P}_{k}}\right) \leq c(2 k-1)^{t n} \quad \text { for all } n \geq 1 .
$$

It is not hard to show [17] that a subset $\mathcal{P}_{k} \subseteq \mathcal{C}_{k}$ is exponentially generic if and only if

$$
\lim _{n \rightarrow \infty} \frac{\#\left\{w \in \mathcal{P}_{k}:|w| \leq n\right\}}{\#\left\{w \in \mathcal{C}_{k}:|w| \leq n\right\}}=1,
$$

with exponentially fast convergence. 
Definition 3.2. Let $k \geq 2$ and $m \geq 1$ be integers. We say that a subset $u_{k, m} \subseteq \mathcal{C}_{k}^{m}$ is generic if

$$
\lim _{n \rightarrow \infty} \frac{\#\left\{\left(r_{1}, \ldots, r_{m}\right) \in \mathcal{U}_{k, m}:\left|r_{i}\right| \leq n, i=1, \ldots, m\right\}}{\#\left\{\left(r_{1}, \ldots, r_{m}\right) \in \mathcal{C}_{k}^{m}:\left|r_{i}\right| \leq n, i=1, \ldots, m\right\}}=1 .
$$

If, in addition, this convergence is exponentially fast, we say that $U_{k, m} \subseteq \ell_{k}^{m}$ is exponentially generic.

It is easy to see that if $\mathcal{P}_{k} \subseteq \mathcal{C}_{k}$ is exponentially generic in $\mathcal{C}_{k}$, then for every $m \geq 1$ the subset $\mathcal{P}_{k}^{m} \subseteq \mathcal{C}_{k}^{m}$ is exponentially generic in $\mathcal{C}_{k}^{m}$. Moreover, it is also not hard to show that in this case for every $m \geq 1$,

$$
\lim _{n \rightarrow \infty} \frac{\#\left\{\left(r_{1}, \ldots, r_{m}\right) \in \mathcal{P}_{k}^{m}:\left|r_{i}\right|=n, i=1, \ldots, m\right\}}{\#\left\{\left(r_{1}, \ldots, r_{m}\right) \in \mathcal{C}_{k}^{m}:\left|r_{i}\right|=n, i=1, \ldots, m\right\}}=1,
$$

with exponentially fast convergence.

Definition 3.3 (Arzhantseva-Ol'shanskii genericity). Let $\mathcal{P}$ be a property of groups.

For integers $k \geq 2, m \geq 1$ we say that a property of groups $\mathcal{P}$ is (exponentially) $(k, m)$-generic if the set $U_{k, m}$ of all $m$-tuples $\left(r_{1}, \ldots, r_{m}\right) \in \mathcal{C}_{k}^{m}$ such that the group $\left\langle a_{1}, \ldots, a_{k} \mid r_{1}, \ldots, r_{m}\right\rangle$ has property $\mathcal{P}$ is an (exponentially) generic subset of $\mathcal{C}_{k}^{m}$.

We say that $\mathcal{P}$ is (exponentially) generic if it is (exponentially) $(k, m)$-generic for every $k \geq 2, m \geq 1$.

\section{Genericity entropy and low-density randomness}

Definition 4.1. Let $\mathscr{P}_{k} \subseteq \mathcal{C}_{k}$ be a set of cyclically reduced words. We define the genericity entropy $t=t\left(\mathcal{P}_{k}\right)$ of $\mathscr{P}_{k}$ as

$$
t:=\limsup _{n \rightarrow \infty} \frac{\log \gamma\left(n, \overline{\mathcal{P}_{k}}\right)}{n \log (2 k-1)} .
$$

We also define the lower genericity entropy $t^{\prime}=t^{\prime}\left(\mathscr{P}_{k}\right)$ as

$$
t^{\prime}:=\liminf _{n \rightarrow \infty} \frac{\log \gamma\left(n, \overline{\mathcal{P}_{k}}\right)}{n \log (2 k-1)} .
$$

It is easy to see that we always have $0 \leq t^{\prime}\left(\mathcal{P}_{k}\right) \leq t\left(\mathcal{P}_{k}\right) \leq 1$ and that $\mathcal{P}_{k} \subseteq \mathcal{C}_{k}$ is exponentially generic if and only if $t\left(\mathcal{P}_{k}\right)<1$.

A simple but crucial computation shows that genericity entropy controls the density parameter in Gromov's model of random groups: 
Proposition 4.2. Let $k \geq 2$ and let $\mathcal{P}_{k} \subseteq \mathcal{C}_{k}$.

(1) Suppose that $t:=t\left(\mathscr{P}_{k}\right)<1$. Let $0<d<1$ be such that $d<1-t$. Then

$$
\lim _{n \rightarrow \infty} \frac{\#(2 k-1)^{d n} \text {-tuples of elements of } \mathcal{P}_{k} \text { of length } n}{\#(2 k-1)^{d n} \text {-tuples of elements of } \mathcal{C}_{k} \text { of length } n}=1 .
$$

(2) Suppose that $d>1-t^{\prime}$ where $t^{\prime}=t^{\prime}\left(\mathcal{P}_{k}\right)$. Then

$$
\lim _{n \rightarrow \infty} \frac{\#(2 k-1)^{d n} \text {-tuples of elements of } \mathcal{P}_{k} \text { of length } n}{\#(2 k-1)^{d n} \text {-tuples of elements of } \mathcal{C}_{k} \text { of length } n}=0 .
$$

Proof. (1) Recall that there exist $0<c_{0}<c_{1}<\infty$ such that for every $n \geq 1$ we have

$$
c_{0}(2 k-1)^{n} \leq \gamma\left(n, \mathscr{C}_{k}\right) \leq c_{1}(2 k-1)^{n} .
$$

Indeed, a result of Rivin [31] shows that

$$
\gamma\left(n, \mathcal{C}_{k}\right)=(2 k-1)^{n}+1+(k-1)\left[1+(-1)^{n}\right] .
$$

Thus for a fixed $k \geq 2$ we have $\gamma\left(n, \boldsymbol{C}_{k}\right) \sim(2 k-1)^{n}$ where $f(n) \sim g(n)$ means that $\lim _{n \rightarrow \infty} \frac{f(n)}{g(n)}=1$.

Let $m=(2 k-1)^{d n}$. The number $N$ of $m$-tuples of elements of $\mathcal{C}_{k}$ of length $n$ where at least one element does not belong to $\mathcal{P}_{k}$ satisfies

$$
\begin{aligned}
\frac{N}{\gamma\left(n, \mathscr{C}_{k}\right)^{m}} & \leq \frac{m \gamma\left(n, \overline{\mathcal{P}_{k}}\right) \gamma\left(n, \mathscr{C}_{k}\right)^{m-1}}{\gamma\left(n, \mathscr{C}_{k}\right)^{m}}=\frac{m \gamma\left(n, \overline{\mathcal{P}_{k}}\right)}{\gamma\left(n, \mathscr{C}_{k}\right)} \\
& \leq \frac{(2 k-1)^{d n} c(2 k-1)^{t n}}{c_{0}(2 k-1)^{n}}=\frac{c(2 k-1)^{(t+d) n}}{c_{0}(2 k-1)^{n}} \underset{n \rightarrow \infty}{\longrightarrow} 0 .
\end{aligned}
$$

This implies part (1) of the proposition.

(2) Again let $m=(2 k-1)^{n d}$. Recall that $d>1-t^{\prime}$, so that $t^{\prime}>1-d$. Let $t^{\prime \prime}$ be such that $t^{\prime}>t^{\prime \prime}>1-d$. Then for $n \gg 1$ we have

$$
\gamma\left(n, \overline{\mathcal{P}_{k}}\right) \geq(2 k-1)^{n t^{\prime \prime}}
$$

and hence

$$
\gamma\left(n, \mathscr{P}_{k}\right)=\gamma\left(n, \mathscr{C}_{k}\right)-\gamma\left(n, \overline{\mathcal{P}_{k}}\right) \leq \gamma\left(n, \mathscr{C}_{k}\right)-(2 k-1)^{n t^{\prime \prime}} .
$$

Thus $\gamma\left(n, \mathcal{P}_{k}\right)^{m}$ is the number of $m$-tuples of elements of $\mathcal{P}_{k}$ of length $n$ and it satisfies

$$
\begin{aligned}
\frac{\gamma\left(n, \mathcal{P}_{k}\right)^{m}}{\gamma\left(n, \mathscr{C}_{k}\right)^{m}} & \leq \frac{\left(\gamma\left(n, \mathscr{C}_{k}\right)-(2 k-1)^{n t^{\prime \prime}}\right)^{m}}{\gamma\left(n, \mathscr{C}_{k}\right)^{m}} \\
& =\left(\frac{\gamma\left(n, \mathscr{C}_{k}\right)-(2 k-1)^{n t^{\prime \prime}}}{\gamma\left(n, \mathscr{C}_{k}\right)}\right)^{m}=\left(1-\frac{(2 k-1)^{n t^{\prime \prime}}}{\gamma\left(n, \mathcal{C}_{k}\right)}\right)^{m}
\end{aligned}
$$


Denote $Y_{n}=\log \frac{\gamma\left(n, \mathcal{P}_{k}\right)^{m}}{\gamma\left(n, \mathscr{C}_{k}\right)^{m}}$. Then

$$
\begin{aligned}
Y_{n} & \leq m \log \left(1-\frac{(2 k-1)^{n t^{\prime \prime}}}{\gamma\left(n, e_{k}\right)}\right)=(2 k-1)^{n d} \log \left(1-\frac{(2 k-1)^{n t^{\prime \prime}}}{\gamma\left(n, \bigodot_{k}\right)}\right) \\
& \sim(2 k-1)^{n d}\left(-\frac{(2 k-1)^{n t^{\prime \prime}}}{\gamma\left(n, \bigodot_{k}\right)}\right) \sim-(2 k-1)^{n d} \frac{(2 k-1)^{n t^{\prime \prime}}}{(2 k-1)^{n}} \\
& =-\frac{(2 k-1)^{n\left(d+t^{\prime \prime}\right)}}{(2 k-1)^{n}}=-\left(\frac{(2 k-1)^{d+t^{\prime \prime}}}{2 k-1}\right)^{n} \underset{n \rightarrow \infty}{\longrightarrow}-\infty,
\end{aligned}
$$

since $d+t^{\prime \prime}>1$. (Recall that $f(n) \sim g(n)$ means that $\lim _{n \rightarrow \infty} \frac{f(n)}{g(n)}=1$.)

Hence $\lim _{n \rightarrow \infty} \log \frac{\gamma\left(n, \mathcal{P}_{k}\right)^{m}}{\gamma\left(n, \mathscr{C}_{k}\right)^{m}}=-\infty$ and therefore $\lim _{n \rightarrow \infty} \frac{\gamma\left(n, \mathcal{P}_{k}\right)^{m}}{\gamma\left(n, \mathscr{C}_{k}\right)^{m}}=0$, as claimed.

Corollary 4.3. For each $k \geq 2$ let $\mathcal{P}_{k} \subseteq \boldsymbol{C}_{k}$. Let $\mathcal{E}=\left(\mathscr{E}_{k}\right)_{k \geq 2}$ where $\mathscr{E}_{k}$ is the property that for a finite presentation on $k$ generators all the defining relations belong to $\mathcal{P}_{k}$. Let $t_{k}=t\left(\mathcal{P}_{k}\right)$ and let $t_{k}^{\prime}=t^{\prime}\left(\mathcal{P}_{k}\right)$. Then the following holds:

(1) If $0 \leq t_{k}<1$ for every $k \geq 2$ then the property $\mathcal{E}$ is monotone low-density random.

(2) If $\sup _{k} t_{k}^{\prime}=1$ then there does not exist $d>0$ such that $\mathcal{E}$ is $d$-random.

\section{Comparing the two models}

The proofs of most existing results related to the Arzhantseva-Ol'shanskii genericity model rely on proving that certain subsets $\mathcal{P}_{k} \subseteq \mathcal{C}_{k}$ are exponentially generic.

We recall the definitions of crucial genericity conditions for many results using the Arzhantseva-Ol'shanskii genericity model.

Definition 5.1 ([5]). Let $0<\mu<1$ and let $k \geq 2$ be an integer. A freely reduced word $w$ in $F\left(a_{1}, \ldots, a_{k}\right)$ is $\mu$-readable if there exists a finite connected graph $\Gamma$, with a distinguished base-vertex, with the following properties:

(1) Every edge $e$ of $\Gamma$ is labelled by some element $s(e)$ of $\left\{a_{1}, \ldots, a_{k}\right\}^{ \pm 1}$ so that for every edge $e$ we have $s\left(e^{-1}\right)=s(e)^{-1}$.

(2) The graph $\Gamma$ is folded, that is, there is no vertex with two distinct edges originating at that vertex and having the same label.

(3) $\Gamma$ has no degree-one vertices except possibly for its base-vertex.

(4) The fundamental group of $\Gamma$ is free of rank at most $k-1$.

(5) There exists an immersed path in $\Gamma$ labelled $w$. 
(6) The volume of $\Gamma$ (that is, the number of non-oriented edges) is at most $\mu|w|$.

We denote by $\mathcal{P}_{k}(\mu)$ the set of all non- $\mu$-readable elements of $\mathcal{C}_{k}$.

Definition 5.2 ([1]). Let $L \geq 2$ and $k \geq 2$ be integers. Let $0<\mu<1$. We say that a freely reduced word $v \in F\left(a_{1}, \ldots, a_{k}\right)$ is $(\mu, L)$-readable if there exists a finite connected graph $\Gamma$ with the following properties:

(1) Every edge $e$ of $\Gamma$ is labelled by some element $s(e)$ of $\left\{a_{1}, \ldots, a_{k}\right\}^{ \pm 1}$ so that for every edge $e$ we have $s\left(e^{-1}\right)=s(e)^{-1}$.

(2) The graph $\Gamma$ is folded.

(3) The fundamental group of $\Gamma$ is free of rank at most $L$.

(4) The graph $\Gamma$ has at least one vertex of degree $<2 k$.

(5) The graph $\Gamma$ has at most two degree-1 vertices.

(6) There exists an immersed path in $\Gamma$ labelled $v$.

(7) The volume of $\Gamma$ (that is, the number of non-oriented edges) is at most $\mu|v|$.

We denote by $\mathcal{Q}_{k}(\mu, L)$ the set of all non- $(\mu, L)$-readable elements of $\mathcal{C}_{k}$.

A key result of [5] is that for fixed $k$ and a sufficiently small $\mu$ (namely, when $\mu<\log _{2 k}\left(1+\frac{1}{4 k-4}\right)$ ) the set of non- $\mu$-readable elements is exponentially generic in $\mathcal{C}_{k}$. Arzhantseva [1] also obtained a similar result regarding non- $(\mu, L)$-readable words:

Proposition 5.3 ([5], [1]). Let $k \geq 2$ be an integer and let $F=F\left(a_{1}, \ldots, a_{k}\right)$. Then the following holds:

(1) Let $0<\mu<\log _{2 k}\left(1+\frac{1}{4 k-4}\right)<1$. Then the set $\mathcal{P}_{k}(\mu)$ of all non- $\mu$-readable elements of $\mathcal{C}_{k}$ is exponentially generic in $\mathcal{C}_{k}$.

(2) Let $L \geq 2$ be and integer and let

$$
0<\mu<\frac{1}{3 L} \log _{2 k}\left(1+\frac{1}{2(2 k-1)^{3 L}-2}\right)<1 .
$$

Then the set $\mathcal{Q}_{k}(\mu, L)$ of all non- $(\mu, L)$-readable words in $\mathcal{C}_{k}$ is exponentially generic in $\mathcal{C}_{k}$.

In most results related to the Arzhantseva-Ol'shanskii genericity one works with intersections of properties that either monotone low-density random (such as conditions involving non- $\mu$-readable words and non- $(\mu, L)$-readable words) or monotone random with some density parameter $d>0$ independent of $k$ (such as the small cancellation condition $C^{\prime}(\lambda)$ for a fixed $0<\lambda<1$ ). Therefore the resulting conditions are in fact monotone low-density random. We give here a summary 
of some statements that follow from the proofs of various known results related to Arzhantseva-Ol'shanskii genericity using Corollary 4.3. At the end of each item we give a reference to the source where the corresponding statement was established in the Arzhantseva-Olshanskii model of genericity.

Theorem 5.4. The following properties are monotone low-density random (where $k$ varies over $k=2, \ldots)$ :

(1) the property that a finite group presentation satisfies the $C^{\prime}(\lambda)$-small cancellation condition (where $0<\lambda \leq 1 / 6$ is any fixed number independent of $k$ ) and defines a group $G$ that is one-ended, torsion-free, and word-hyperbolic [5];

(2) the property that a finite presentation on generators $a_{1}, \ldots, a_{k}$ defines a group $G$ such that all $(k-1)$-generated subgroups are free and quasiconvex in $G$ [5];

(3) the property that a finite presentation on generators $a_{1}, \ldots, a_{k}$ defines a group $G$ with $\operatorname{rk}(G)=k[5]$;

(4) the property that a finite presentation on generators $a_{1}, \ldots, a_{k}$ defines a group $G$ such that all $L_{k}$-generated subgroups of infinite index in $G$ are free and quasiconvex in $G$ (here $L_{k}$ is any sequence of positive integers) [1], [2];

(5) the property that for a $k$-generated finitely presented group $G$ there is exactly one Nielsen-equivalence class of $k$-tuples of elements generating non-free subgroups [18].

Regarding condition (1) in Theorem 5.4, it is known that the property of a finitely presented group to be non-elementary torsion-free word-hyperbolic is in fact monotone random and not just low-density random (see Theorem 2 in [24], Theorem 11 in [27]). It is also known and not hard to prove that if $0<\lambda<1$ and $0<d<\lambda / 2$ then the $C^{\prime}(\lambda)$ small cancellation condition is a monotone $d$-random property (see, for example, Proposition 10 of [27]).

Unlike the case of the standard small cancellation condition, the genericity entropy $t$ for exponentially generic sets arising from the Arzhantseva-Ol'shanskii nonreadability conditions usually depends on $k$ and in fact converges to 1 as $k \rightarrow \infty$. This situation is different from the standard small cancellation conditions where the genericity entropy is easily seen to have a positive upper bound which is separated from 1 and independent of $k$. In Section 6 we establish this for the non- $\mu$-readability condition and show that in that case $\lim _{k \rightarrow \infty} t^{\prime}\left(\mathcal{P}_{k}(\mu)\right)=\lim _{k \rightarrow \infty} t\left(\mathscr{P}_{k}(\mu)\right)=1$. Hence for $0<d(k)<1-t^{\prime}\left(\mathcal{P}_{k}\right)$ we have $\lim _{k \rightarrow \infty} d(k)=0$ and, in view of Corollary 4.3 , the notion of low-density randomness becomes necessary. 


\section{Detailed examples of low-density random but not random properties}

Proposition 6.1. Let $k \geq 2$ and let $0<\mu_{k}<1$. Let $\mathcal{P}_{k}\left(\mu_{k}\right) \subseteq \boldsymbol{\varphi}_{k}$ be the set of all cyclically reduced words in $F\left(a_{1}, \ldots, a_{k}\right)$ that are not $\mu_{k}$-readable. Then

$$
1 \geq t\left(\mathscr{P}_{k}\right) \geq t^{\prime}\left(\mathscr{P}_{k}\right) \geq \frac{\log (2 k-3)}{\log (2 k-1)}
$$

and hence

$$
\lim _{k \rightarrow \infty} t\left(\mathcal{P}_{k}\right)=\lim _{k \rightarrow \infty} t^{\prime}\left(\mathcal{P}_{k}\right)=1
$$

Proof. Let $\Gamma$ be the wedge of $(k-1)$ loop-edges labelled by $a_{1}, \ldots, a_{k-1}$. Then any freely reduced word from $F\left(a_{1}, \ldots, a_{k-1}\right)$ can be read as the label of a path in $\Gamma$. Hence for any $w \in \ell_{k-1}$ with $|w|>(k-1) / \mu_{k}$ the word $w$ is $\mu_{k}$-readable, that is, $w \in \overline{\mathcal{P}_{k}}$. Thus for $n \geq 1+\mu_{k}^{-1}(k-1)$ we have

$$
\gamma\left(n, \overline{\mathcal{P}_{k}}\right) \geq \gamma\left(n, \bigodot_{k-1}\right) \geq c(2 k-3)^{n}
$$

for some constant $c>0$ independent of $n$.

Therefore

$$
t^{\prime}\left(\mathscr{P}_{k}\right) \geq \liminf _{n \rightarrow \infty} \frac{\log c(2 k-3)^{n}}{n \log (2 k-1)}=\frac{\log (2 k-3)}{\log (2 k-1)}
$$

as claimed.

Part (2) of Corollary 4.3 immediately implies

Corollary 6.2. Let $0<\mu_{k}<1$ for $k \geq 2$. Let $\mathcal{E}=\left(\mathscr{G}_{k}\right)_{k \geq 2}$, where $\mathscr{G}_{k}$ is the property that for a finite presentation on $k$ generators all the defining relations are non- $\mu_{k}$-readable.

Then $\mathcal{G}$ is not $d$-random for any $d>0$.

Similarly, one obtains the following result.

Corollary 6.3. Let $\mathcal{G}=\left(\mathscr{E}_{k}\right)_{k \geq 2}$, where $\mathscr{E}_{k}$ is the property that a finite group presentation on the generators $a_{1}, \ldots a_{k}$ defines a group $G$ such that every proper subset of $a_{1}, \ldots a_{k}$ freely generates a free subgroup of $G$.

Then $\mathcal{G}$ is monotone low-density random but not $d$-random for any $d>0$.

Proof. The fact that $\mathcal{G}$ is not $d$-random for any $d>0$ follows from part (2) of Corollary 4.3 by the same argument as in the proof of Proposition 6.1.

It is well known (see, for example, [27], Proposition 10) that the $C^{\prime}(\lambda)$ small cancellation condition is a monotone $d$-random property for any $0<d<\lambda / 2$. It 
is also easy to see that the set of cyclically reduced words $r$ in $\ell_{k}$ such that every subword of $r$ of length $|r| / 6$ involves all the generators $a_{1}, \ldots, a_{k}$ is exponentially generic in $\ell_{k}$. Let $G$ be given by a $C^{\prime}(1 / 6)$-presentation on the generators $a_{1}, \ldots, a_{k}$ where all the defining relations $r$ have the property that every subword of $r$ of length $|r| / 6$ involves all the generators $a_{1}, \ldots, a_{k}$. Then every proper subset of $a_{1}, \ldots, a_{k}$ freely generates a subgroup of $G$. It now follows from part (1) of Corollary 4.3 that $\mathcal{P}$ is monotone low-density random.

One can regard property $\mathcal{E}$ from Corollary 6.3 above as a version of Magnus' Freiheitssatz for random groups. An asymptotic version of the Freiheitssatz using another model introduced by Gromov [12] was obtained by Cherix and Schaeffer [10].

Similar arguments to those used above yield

Proposition 6.4. Let $\mathcal{G}=\left(\mathscr{E}_{k}\right)_{k \geq 2}$ where $\mathscr{E}_{k}$ is the property that a finite group presentation on $a_{1}, \ldots, a_{k}$ defines a group of rank $k$. Then $\mathcal{E}$ is monotone low-density random but not $d$-random for any $d>0$.

Proof. We have already observed in Theorem 5.4 that $\mathscr{G}$ is monotone low-density random. Let $\mathscr{G}^{\prime}=\left(\mathscr{G}_{k}^{\prime}\right)_{k \geq 2}$ where $\mathscr{E}_{k}^{\prime}$ is the property that for a finite presentation on $a_{1}, \ldots, a_{k}$ none of the defining relations are primitive in $F\left(a_{1}, \ldots, a_{k}\right)$. Clearly, if $G=\left\langle a_{1}, \ldots, a_{k} \mid r_{1}, \ldots, r_{m}\right\rangle$ and some $r_{i}$ is a primitive element in $F\left(a_{1}, \ldots, a_{k}\right)$ (that is, $r_{i}$ belongs to some free basis of $F\left(a_{1}, \ldots, a_{k}\right)$ ) then $\operatorname{rk}(G) \leq k-1$. Thus $\mathscr{G}_{k} \subseteq \mathscr{G}_{k}^{\prime}$ and $\mathscr{G} \subseteq \mathscr{G}^{\prime}$. It suffices to show that $\mathscr{G}^{\prime}$ is not $d$-random for any $d>0$.

Let $\mathscr{P}_{k} \subseteq \mathcal{C}_{k}$ be the set of all non-primitive elements in $\mathcal{C}_{k}$. Note that, for any freely reduced word $w \in F\left(a_{2}, \ldots a_{k}\right)$, the element $a_{1} w$ is primitive in $F\left(a_{1}, \ldots, a_{k}\right)$. Hence

$$
\gamma\left(n, \overline{\mathcal{P}_{k}}\right) \geq \gamma\left(n-1, F_{k-1}\right)=(2 k-2)(2 k-3)^{n-2} .
$$

Therefore

$$
1 \geq t\left(\mathcal{P}_{k}\right) \geq t^{\prime}\left(\mathcal{P}_{k}\right) \geq \frac{\log (2 k-3)}{\log (2 k-1)} \underset{k \rightarrow \infty}{\longrightarrow} 1 .
$$

Part (2) of Corollary 4.3 implies that $\mathcal{E}^{\prime}$ is not $d$-random for any $d>0$.

\section{A bounded freeness property}

In this section we will show that for every fixed integer $L \geq 2$ there is some $0<d<1$ such that the property of a finitely presented group that all its $L$-generated subgroups of infinite index are free is monotone $d$-random.

First, we need to investigate the genericity entropy of the set of non- $(\mu, L)$-readable words. Recall that $Q_{k}(\mu, L)$ is the set of all words in $\ell_{k}$ that are not $(\mu, L)$ readable. The proof of the following proposition is similar to the counting arguments 
used in [1], [5], with a variation whose significance is explained further in Remark 7.6 below.

Proposition 7.1. Let $k \geq 2$ be a fixed integer and let $2 \leq L<k$. Then we have

$$
\gamma\left(n, \overline{Q_{k}(\mu, L)}\right) \leq C(\mu n)^{3 L+1}(6 L)^{n}(2 k-1)^{\mu n} .
$$

where $C>0$ is independent of $n$.

Proof. Recall that an arc in $\Gamma$ is an immersed edge-path where every intermediate vertex of the path has degree two in $\Gamma$.

Note that if $\Gamma$ is a finite connected graph with fundamental group free of rank $\leq L<k$, then $\Gamma$ necessarily has a vertex of degree $<2 k$. Thus condition (4) of Definition 5.2 is redundant in this case.

Let $L>k$ and $0<\mu<1$ be fixed. Let $v \in F\left(a_{1}, \ldots, a_{k}\right)$ be a $(\mu, L)$ readable word with $|v|=n$. First, we estimate the number of labelled graphs $\Gamma$ as in Definition 5.2 where $v$ can be read.

There are $\leq C_{0}=C_{0}(L)$ topological types of the graphs $\Gamma$ arising in the definition of a $(\mu, L)$-readable word. Since $\pi_{1}(\Gamma)$ has rank at most $L$ and $\Gamma$ has at most two degree- 1 vertices, it follows that $\Gamma$ has $\leq 3 L$ non-directed maximal arcs and $\leq 6 L$ directed maximal arcs. The sum of the length of theses arcs is $\leq \mu n$. The number of ways to represent a positive integer $N$ as a sum

$$
N=N_{1}+\cdots+N_{3 L},
$$

where $N_{i}$ are non-negative integers is

$$
\frac{(N+3 L-1) !}{N !(3 L-1) !} \leq(N+3 L-1)^{3 L} .
$$

Hence the number of ways to write a sum

$$
N_{1}+\cdots+N_{3 L} \leq \mu n
$$

is $\leq C_{1}(\mu n)^{3 L+1}$, where $C_{1}$ is independent of $n$. Now for each decomposition $N_{1}+\cdots+N_{3 L} \leq \mu n$, the number of ways to assign the maximal arcs of $\Gamma$ labels $v_{1}, \ldots v_{3 L} \in F\left(a_{1}, \ldots, a_{k}\right)$ with $\left|v_{i}\right|=N_{i}$ is

$$
\leq C_{2}(2 k-1)^{\mu n} \text {, }
$$

where $C_{2}>0$ does not depend on $n$.

Thus there are at most $C_{0} C_{1} C_{2}(\mu n)^{3 L+1}(2 k-1)^{\mu n}$ relevant labelled graphs $\Gamma$ as in Definition 5.2

For a fixed $\Gamma$, if $v$ can be read in $\Gamma$ then $v$ is the label of a path

$$
p_{1}^{\prime}, p_{2}, \ldots, p_{s-1}, p_{s}^{\prime}
$$


where $p_{i}$ are oriented maximal arcs, $p_{1}^{\prime}, p_{s}^{\prime}$ are oriented arcs and $s \leq|v|=n$. By passing to a subgraph of $\Gamma$ if necessary we may assume that $p_{1}^{\prime}$ and $p_{2}^{\prime}$ are maximal arcs as well. Thus $v$ is the label of a path $\alpha=p_{1}, p_{2}, \ldots, p_{s-1}, p_{s}$ where $p_{i}$ are directed maximal arcs in $\Gamma$ and where $s \leq n=|v|$. Since $s \leq n$ and $\Gamma$ has $\leq 6 L$ oriented maximal arcs, there are $\leq(6 L)^{n}$ combinatorial possibilities to express $\alpha$ as a word in the alphabet of $6 L$ letters corresponding to the directed maximal arcs.

Hence the total number of possibilities for $v$ is

$$
\gamma\left(n, \overline{Q_{k}(\mu, L)}\right) \leq C_{0} C_{1} C_{2}(\mu n)^{3 L+1}(6 L)^{n}(2 k-1)^{\mu n},
$$

as required.

The following technical definition is motivated by the corresponding notions used in counting arguments in [1], [5].

Definition 7.2. Let $k \geq 2, L \geq 2$ be integers and let $0<\mu<1$. We say that a cyclically reduced word $w \in F\left(a_{1}, \ldots, a_{k}\right)$ is $(\mu, L)$-good if no cyclic permutation of $w^{ \pm 1}$ contains a subword $v$ of length $\geq|w| / 2$ such that $v$ is $(\mu, L)$-readable.

Lemma 7.3. Let $k>L \geq 2$ and let $0<\mu<1$. Let $y_{k}=y_{k}(\mu, L) \subseteq \bigodot_{k}$ be the set of all cyclically reduced $(\mu, L)$-good words. Then

$$
t\left(y_{k}\right) \leq \frac{((\mu+1) / 2) \log (2 k-1)+(1 / 2) \log (6 L)}{\log (2 k-1)} .
$$

Proof. Let $w \in \overline{y_{k}}$ with $n=|w|$. There are at most $2 n$ cyclic permutations of $w^{ \pm 1}$ and at least one of them has an initial segment $v$ of length $n / 2$ such that $v$ is $(\mu, L)$-readable. Hence by Proposition 7.1 the number of possibilities for $w$ is

$$
\gamma\left(n, \overline{y_{k}}\right) \leq A(2 n)(\mu n / 2)^{3 L+1}(6 L)^{n / 2}(2 k-1)^{\mu n / 2}(2 k-1)^{n / 2},
$$

where $A>0$ is independent of $n$. Hence

$$
\begin{aligned}
t\left(y_{k}\right) & =\limsup _{n \rightarrow \infty} \frac{\log \gamma\left(n, \overline{y_{k}}\right)}{n \log (2 k-1)} \\
& \leq \limsup _{n \rightarrow \infty} \frac{\left(\frac{n}{2}+\frac{\mu n}{2}\right) \log (2 k-1)+\frac{n}{2} \log 6 L+\log (2 A n)+(3 L+1) \log \frac{\mu n}{2}}{n \log (2 k-1)} \\
& =\frac{\left(\frac{\mu+1}{2}\right) \log (2 k-1)+\frac{1}{2} \log (6 L)}{\log (2 k-1)} .
\end{aligned}
$$

The results of Section 4 of [1] imply 
Proposition 7.4. Let $L, k \geq 2$ be integers. Let $0<\mu<1$ and $0<\lambda<1$ be such that

$$
0<\lambda \leq \frac{\mu}{15 L+3 \mu} \leq \frac{1}{6} .
$$

Let $G=\left\langle a_{1}, \ldots, a_{k} \mid r_{1}, \ldots, r_{m}\right\rangle$ be such that

(1) the above presentation of $G$ satisfies the small cancellation condition $C^{\prime}(\lambda)$,

(2) all $r_{1}, \ldots, r_{m}$ are cyclically reduced words that are not proper powers in $F\left(a_{1}, \ldots, a_{k}\right)$, and

(3) each $r_{i}$ is $(\mu, L)$-good.

Then every L-generated subgroup of infinite index in $G$ is free.

Theorem 7.5. For every integer $L \geq 2$ there is some $d>0$ such that the property of finitely presented groups for all L-generated subgroups of infinite index to be free is monotone d-random.

Proof. Let $L \geq 2$ be a fixed integer.

It is well known and easy to see that conditions (1) and (2) from Proposition 7.4 are monotone random (see, e.g., Proposition 10 and Theorem 11 in [27]). Thus it suffices to deal with condition (3) of Proposition 7.4.

Choose $0<\lambda, \mu<1$ so that

$$
0<\lambda \leq \frac{\mu}{15 L+3 \mu} \leq \frac{1}{6}
$$

We have

$$
\lim _{k \rightarrow \infty} \frac{((\mu+1) / 2) \log (2 k-1)+(1 / 2) \log (6 L)}{\log (2 k-1)}=\frac{\mu+1}{2}<1 .
$$

Choose $v$ so that $(\mu+1) / 2<v<1$. There exists an integer $k_{0}>L$ such that

$$
\frac{((\mu+1) / 2) \log (2 k-1)+(1 / 2) \log (6 L)}{\log (2 k-1)} \leq v<1
$$

for any $k \geq k_{0}$. Thus by Lemma 7.3 for $k \geq k_{0}$ we have

$$
t\left(y_{k}\right) \leq v<1 .
$$

Recall that by Theorem 5.4 the property of having all $L$-generated subgroups of infinite index being free is monotone low-density random with density sequence $(d(k))_{k \geq 2}$. Put $d_{0}:=\min \left\{d(2), \ldots, d\left(k_{0}-1\right), 1-v\right\}$. Then by Proposition 4.2 the property of having all $L$-generated subgroups of infinite index being free is monotone $d$-random for any $0<d<d_{0}$. 
Remark 7.6. In [1] Arzhantseva presented a proof of exponential genericity in $F\left(a_{1}, \ldots, a_{k}\right)$ of non- $(\mu, L)$-readable words, assuming that $\mu$ is small enough. However, the estimates on the growth of $(\mu, L)$-readable words obtained there are insufficient for our purposes in the proof of Theorem 7.5. Let $\mathcal{P}_{k} \subseteq \mathcal{C}_{k}$ be the set of all non- $\left(\mu_{k}, L\right)$-readable cyclically reduced words in $F\left(a_{1}, \ldots, a_{k}\right)$, where $0<\mu_{k}<1$ satisfies

$$
0<\mu_{k}<\frac{1}{3 L} \log _{2 k}\left(1+\frac{1}{2(2 k-1)^{3 L}-2}\right) .
$$

A crucial estimate in Lemma 3 of [1] shows that

$$
\gamma\left(n, \overline{\mathcal{P}_{k}}\right) \leq A\left((2 k-1)^{3 L}-\frac{1}{2}\right)^{n / 3 L} \text {. }
$$

This yields

$$
t\left(\mathcal{P}_{k}\right) \leq \frac{\log \left((2 k-1)^{3 L}-\frac{1}{2}\right)}{3 L \log (2 k-1)} \underset{k \rightarrow \infty}{\longrightarrow} 1,
$$

where convergence to 1 in the last limit is easily seen by applying l'Hôpital's rule. Therefore we needed an estimate different from $(* *)$ for the number of $(\mu, L)$ readable words in Proposition 7.1. That estimate allowed us to obtain bounds on the genericity entropy of the set of $(\mu, L)$-good words that are independent of $k$ for sufficiently large $k$. On the other hand, we still needed the results of [1] obtained via the estimate $(* *)$ to deal with the case of "small" $k$ with $k<k_{0}$ in the proof of Theorem 7.5.

\section{Double-exponential lower bound for $J_{\epsilon}(t)$}

In this section we establish Theorem 1.3 from the Introduction (see Theorem 8.2 below). Note that Theorem 1.3 implies Corollary 1.4 giving Erschler's doubleexponential lower bound for the number $I_{2}(n)$ of isomorphism types of quotients of $F(a, b)$ by collections of defining relations of length $\leq n$.

It is well known that the modular group $\operatorname{PSL}(2, \mathbb{Z})$ is isomorphic to the free product of a cyclic group of order two and a cyclic group of order three. Denote

$$
M:=\left\langle a, b \mid a^{2}=b^{3}=1\right\rangle=\left\langle a \mid a^{2}=1\right\rangle *\left\langle b \mid b^{3}=1\right\rangle .
$$

Put $A=\left\{a, b, b^{-1}\right\}$. We say that a word $w \in A^{*}$ is reduced if it does not contain subwords of the form $a a, b b, b^{-1} b^{-1}, b b^{-1}, b^{-1} b$. it is clear that any element of $M$ is uniquely represented by a reduced word in $A^{*}$. We say that a word $w \in A^{*}$ is cyclically reduced if $w$ and all cyclic permutations of $w$ are reduced. Thus any nonempty cyclically reduced word is either a single letter or, up to a cyclic permutation, has the form

$$
w=a b^{\epsilon_{1}} a b^{\epsilon_{2}} \ldots a b^{\epsilon_{t}}
$$


where $\epsilon_{i}= \pm 1$. It is therefore easy to see that the number of all cyclically reduced words in $A^{*}$ of length $n>1$ is equal to 0 if $n$ is odd, and is equal to $2 \cdot 2^{n / 2}$ if $n$ is even. As before, let $\mathcal{C}_{A}$ be the set of all cyclically reduced words in $A^{*}$. For a subset $S \subseteq \mathcal{C}_{A}$ denote by $\gamma(n, S)$ the number of elements of length $n$ in $\mathcal{C}_{A}$. Similarly to the free group case, we can define the notions of generic and exponentially generic subsets of $\mathcal{C}_{A}$. Thus $S \subseteq \mathcal{C}_{A}$ is exponentially generic if

$$
\lim _{t \rightarrow \infty} \frac{\gamma(2 t, S)}{\gamma\left(2 t, \mathscr{C}_{A}\right)}=\lim _{t \rightarrow \infty} \frac{\gamma(2 t, S)}{2^{t+1}}=1,
$$

with exponentially fast convergence. Similarly, all the other notions of genericity in the Arzhantseva-Ol'shanskii model can be defined for quotients of $M$ in exactly the same way as for the quotients of $F\left(a_{1}, \ldots, a_{k}\right)$.

Denote by $\eta: M \rightarrow M$ the relabelling automorphism of $M$ defined on the generators as $\eta(a)=a, \eta(b)=b^{-1}$.

Notation 8.1. For $\epsilon>0$ be fixed. For an integer $t \geq 1$ let $J_{\epsilon}(t)$ be the number of isomorphism types of groups given by presentations of the form

$$
G=M /\left\langle\left\langle r_{1}, \ldots, r_{m}\right\rangle\right\rangle,
$$

where $m=2^{t \epsilon}$ and where each $r_{i}$ is a cyclically reduced word of length $2 t$ in $A^{*}$.

Theorem 8.2. There exists $\epsilon_{0}>0$ such that for any $0<\epsilon \leq \epsilon_{0}$ there is some $\rho>1$ such that

$$
J_{\epsilon}(t) \geq \rho^{\rho^{t}} \text { for } t \rightarrow \infty,
$$

that is, $J_{\epsilon}(t)$ is bounded below by a double-exponential function of $t$ as $t \rightarrow \infty$.

Proof. The results of [20] show that there is some exponentially generic subset $S \subseteq$ $A^{*}$ and some $0<\lambda<1$ with the following property. Suppose $m \geq 1$ is fixed. Then there exists an exponentially generic subset $U_{m} \subseteq \mathcal{C}_{A}^{m}$ such that:

(1) Every presentation (\$) with $\left(r_{1}, \ldots, r_{m}\right) \in U_{m}$ satisfies the $C^{\prime}(\lambda)$ small cancellation condition.

(2) We have $U_{m} \subseteq S^{m}$.

(3) For $\left(r_{1}, \ldots, r_{m}\right),\left(s_{1}, \ldots, s_{m}\right) \in U_{m}$ with $\left|r_{i}\right|=\left|s_{j}\right|=2 t$ the groups $M /\left\langle\left\langle r_{1}, \ldots, r_{m}\right\rangle\right\rangle$ and $\left.M /\left\langle s_{1}, \ldots, s_{m}\right\rangle\right\rangle$ are isomorphic if and only if there is a reordering $\left(r_{1}^{\prime}, \ldots, r_{m}^{\prime}\right)$ of $\left(r_{1}, \ldots, r_{m}\right)$ and there is $\delta \in\{0,1\}$ such that each $r_{i}^{\prime}$ is a cyclic permutation of $\eta^{\delta}\left(s_{i}\right)$ or of $\eta^{\delta}\left(s_{i}^{-1}\right)$.

(4) The number $K_{m}(t)$ of isomorphism types of groups given by presentation ( + ) where all $r_{i}$ are cyclically reduced words of length $2 t$ in $A^{*}$ satisfies

$$
K_{m}(t) \sim \frac{2^{m(t+1)}}{2 m !(4 t)^{m}} .
$$


Statement (4) is essentially a corollary of (3): one needs to count the number of all presentations $(\$)$ where $\left(r_{1}, \ldots, r_{m}\right) \in U_{m}$ has $\left|r_{1}\right|=\cdots=\left|r_{m}\right|=2 t$ and divide this number by the multiplicity constant in counting the isomorphism types of such presentations, where this multiplicity constant comes from (3) and is equal to $2 m !(4 t)^{m}$. Here the factor $m$ ! comes from counting reorderings $\left(r_{1}^{\prime}, \ldots, r_{m}^{\prime}\right)$ of $\left(r_{1}, \ldots, r_{m}\right) \in U_{m}$. Every $r_{i}$ of length $2 t$ has $2 t$ cyclic permutations, so there are $4 t$ cyclic permutations of $r_{i}^{ \pm 1}$. Finally, applying $\eta^{\delta}$, with $\delta=0,1$, to the presentation gives an additional multiplicity factor of 2 .

The results of the present paper, namely an appropriately adapted version of Corollary 4.3, imply that statements (1)-(3) also hold in the low-density model, where the number of relations $m$ is not fixed but rather has the form $m=2^{t \epsilon}$ with $\epsilon>0$ sufficiently small and independent of $t$. Note that since $S \subseteq \mathcal{C}_{A}$ is exponentially generic, we have $\gamma(2 t, S) \geq \frac{1}{2} \gamma\left(2 t, \mathcal{C}_{A}\right)$ for all sufficiently large $t$. Then the same arguments as in [20] imply that the number $J_{\epsilon}(t)$ satisfies

$$
J_{\epsilon}(t) \geq C \frac{2^{m(t+1)} 2^{-m}}{2 m !(4 t)^{m}}
$$

where $C>0$ is a constant and where $m=2^{t \epsilon}$. It is not hard to see that this gives a double-exponential lower bound for $J_{\epsilon}(t)$. Indeed, note that $m ! \leq m^{m}$ and thus

$$
J_{\epsilon}(t) \geq C \frac{\left(2^{(t+1)}\right)^{m}}{2(8 m t)^{m}} \geq C \frac{\left(2^{(t+1)}\right)^{m}}{(16 m t)^{m}}
$$

hence

$$
\log J_{\epsilon}(t) \geq \log C+m \log \left(\frac{2^{(t+1)}}{16 m t}\right)=\log C+2^{t \epsilon} \log \left(\frac{2^{(t+1)}}{16 \cdot 2^{t \epsilon} t}\right) .
$$

If $\epsilon>0$ is chosen sufficiently small, then

$$
\frac{2^{(t+1)}}{16 \cdot 2^{t \epsilon} t} \geq 2 \quad \text { for } t \rightarrow \infty
$$

and hence

$$
\log J_{\epsilon}(t) \geq \log C+2^{t \epsilon} \log 2 \geq 2^{t \epsilon / 2} \quad \text { for } t \rightarrow \infty,
$$

yielding a double-exponential lower bound for $J_{\epsilon}(t)$, as required.

\section{References}

[1] G. N. Arzhantseva, On groups in which subgroups with a fixed number of generators are free. Fundam. Prikl. Mat. 3 (1997), 675-683. Zbl 0929.20025 MR 1794135 
[2] G. N. Arzhantseva, Generic properties of finitely presented groups and Howson's theorem. Comm. Algebra 26 (1998), 3783-3792. Zbl 0911.20027 MR 1647075

[3] G. N. Arzhantseva, A property of subgroups of infinite index in a free group. Proc. Amer. Math. Soc. 128 (2000), 3205-3210. Zbl 0976.20014 MR 1694447

[4] G. N. Arzhantseva and P.-A. Cherix, On the Cayley graph of a generic finitely presented group. Bull. Belg. Math. Soc. Simon Stevin 11 (2004), 589-601. Zbl 1069.05038 MR 2115727

[5] G. N. Arzhantseva and A. Yu. Ol'shanskiı̌, The class of groups all of whose subgroups with lesser number of generators are free is generic. Mat. Zametki 59 (1996), 489-496; English transl. Math. Notes 59 (1996), 350-355. Zbl 0877.20021 MR 1445193

[6] Ch. Champetier, Propriétés génériques des groupes de présentation finie, $\mathrm{PhD}$ Thesis, Université de Lyon 1, Lyon 1991.

[7] C. Champetier, Propriétés statistiques des groupes de présentation finie. Adv. Math. 116 (1995), 197-262. Zbl 0847.20030 MR 1363765

[8] C. Champetier, L'espace des groupes de type fini. Topology 39 (2000), 657-680. Zbl 0959.20041 MR 1760424

[9] C. Champetier and V. Guirardel, Limit groups as limits of free groups. Israel J. Math. 146 (2005), 1-75. Zbl 1103.20026 MR 2151593

[10] P.-A. Cherix and G. Schaeffer, An asymptotic Freiheitssatz for finitely generated groups. Enseign. Math. (2) 44 (1998), 9-22. Zbl 0987.20012 MR 1643258

[11] É. Ghys, Groupes aléatoires (d'après Misha Gromov,...). Astérisque 294 (2004), 173-204. Zbl 1134.20306 MR 2111644

[12] M. Gromov, Hyperbolic groups. In Essays in group theory, Math. Sci. Res. Inst. Publ. 8, Springer-Verlag, New York 1987, 75-263. Zbl 0634.20015 MR 0919829

[13] M. Gromov, Geometric group theory (Sussex, 1991), vol. 2: Asymptotic invariants of infinite groups. London Math. Soc. Lecture Note Ser. 182, Cambridge University Press, Cambridge 1993. Zbl 0841.20039 MR 1253544

[14] M. Gromov, Random walk in random groups. Geom. Funct. Anal. 13 (2003), 73-146. Zbl 1122.20021 MR 1978492

[15] V. S. Guba, Conditions under which 2-generated subgroups in small cancellation groups are free. Izv. Vyssh. Uchebn. Zaved. Mat. 1986, no. 7, 12-19; English transl. Soviet Math. 30 (1986), no. 7, 14-24. Zbl 0614.20022 MR 867603

[16] V. Kaimanovich, I. Kapovich, and P. Schupp, The subadditive ergodic theorem and generic stretching factors for free group automorphisms. Israel J. Math. 157 (2007), 1-46. Zbl 05145469 MR 2342439

[17] I. Kapovich, A. Miasnikov, P. Schupp, and V. Shpilrain, Generic-case complexity, decision problems in group theory, and random walks. J. Algebra 264 (2003), 665-694. Zbl 1041.20021 MR 1981427

[18] I. Kapovich and P. Schupp, Genericity, the Arzhantseva-Ol'shanskii method and the isomorphism problem for one-relator groups. Math. Ann. 331 (2005), 1-19. Zbl 1080.20029 MR 2107437 
[19] I. Kapovich and P. Schupp, Delzant's T-invariant, Kolmogorov complexity and onerelator groups. Comment. Math. Helv. 80 (2005), 911-933. Zbl 02242667 MR 2182705

[20] I. Kapovich and P. Schupp, Random quotients of the modular group are rigid and essentially incompressible. J. Reine Angew. Math., to appear.

[21] I. Kapovich, P. Schupp, and V. Shpilrain, Generic properties of Whitehead's algorithm and isomorphism rigidity of random one-relator groups. Pacific J. Math. 223 (2006), 113-140. Zbl 05129573 MR 2221020

[22] V. D. Mazurov(ed.), The Kourovka notebook. Unsolved problems in group theory. Including archive of solved problems. 11th ed., Institute of Mathematics, Novosibirsk 1990. Zbl 0748.20001 MR 1176762

[23] Y. Ollivier, Critical densities for random quotients of hyperbolic groups. C. R. Math. Acad. Sci. Paris 336 (2003), 391-394. Zbl 1050.20048 MR 1979351

[24] Y. Ollivier, Sharp phase transition theorems for hyperbolicity of random groups. Geom. Funct. Anal. 14 (2004), 595-679. Zbl 1064.20045 MR 2100673

[25] Y. Ollivier, Cogrowth and spectral gap of generic groups. Ann. Inst. Fourier (Grenoble) 55 (2005), 289-317. Zbl 1133.20032 MR 2141699

[26] Y. Ollivier, Effondrement de quotients aléatoires de groupes hyperboliques avec torsion. C. R. Math. Acad. Sci. Paris 341 (2005), 137-140. Zbl 1077.20057 MR 2158833

[27] Y. Ollivier, A January 2005 invitation to random groups. Ensaios Matemáticos 10, Sociedade Brasileira de Matemática, Rio de Janeiro 2005. Zbl 05012619 MR 2205306

[28] Y. Ollivier and D. T. Wise, Cubulating groups at density 1/6. Preprint 2005. http://www.umpa.ens-lyon.fr/ yollivie/publs.html

[29] Y. Ollivier and D. T. Wise, Kazhdan groups with infinite outer automorphism group. Trans. Amer. Math. Soc. 359 (2007), 1959-1976. Zbl 05120622 MR 2276608

[30] A. Yu. Ol'shanskii, Almost every group is hyperbolic. Internat. J. Algebra Comput. 2 (1992), 1-17. Zbl 0779.20016 MR 1167524

[31] I. Rivin, Growth in free groups (and other stories). Preprint 1999. arXiv:math/9911076

[32] L. Silberman, Addendum to "Random walk on random groups" by M. Gromov Geom. Funct. Anal. 13 (2003), 147-177. Zbl 1124.20027 MR 1978493

[33] A. Żuk, Property (T) and Kazhdan constants for discrete groups. Geom. Funct. Anal. 13 (2003), 643-670. Zbl 1036.22004 MR 1995802

Received May 31, 2007; revised May 27, 2008

I. Kapovich, Department of Mathematics, University of Illinois at Urbana-Champaign, 1409 West Green Street, Urbana, IL 61801, U.S.A.

E-mail: kapovich@math.uiuc.edu

P. Schupp, Department of Mathematics, University of Illinois at Urbana-Champaign, 1409

West Green Street, Urbana, IL 61801, U.S.A.

E-mail: schupp@math.uiuc.edu 